\title{
Biology and control of nausea and vomiting: outcomes of the 2013 University of Pittsburgh conference
}

\author{
Charles C. Horn · Bill J. Yates
}

Published online: 3 July 2014

(C) Springer-Verlag Berlin Heidelberg 2014

A variety of triggers can elicit nausea and vomiting, including ingested toxins, particular drugs such as anesthetics and cancer therapeutics, pregnancy, radiation, and motion that produces unexpected sensory inputs (Stern et al. 2011). Some individuals also suffer from cyclic vomiting syndrome, an under-recognized and often misdiagnosed condition that results in recurring attacks of nausea and vomiting without any apparent triggering mechanism (Lee et al. 2012). It is believed that common neural mechanisms mediate nausea and vomiting, despite the etiology, but there is considerable lack of insight into these mechanisms.

\section{C. Horn}

Biobehavioral Oncology Program, University of Pittsburgh

Cancer Institute, Pittsburgh, PA, USA

\section{C. Horn}

Division of Gastroenterology, Hepatology, and Nutrition, Department of Medicine, University of Pittsburgh School of Medicine, Pittsburgh, PA, USA

\section{C. Horn}

Department of Anesthesiology, University of Pittsburgh School of Medicine, Pittsburgh, PA, USA

C. C. Horn · B. J. Yates

Center for Neuroscience, University of Pittsburgh, Pittsburgh, PA, USA

\section{B. J. Yates $(\bowtie)$}

Department of Otolaryngology, University of Pittsburgh School of Medicine, Eye and Ear Institute, Rm. 110, Pittsburgh,

PA 15213, USA

e-mail: byates@pitt.edu

URL: http://neuroyates.com
Scientists interested in the biology of nausea and vomiting, as well as physicians who treat patients whose lives are negatively affected by these conditions, work in many different academic and clinical departments. Consequently, they attend a variety of professional conferences and publish their research in diverse discipline-specific journals, which has constrained communication in the field. This lack of communication has limited progress in understanding the biological mechanisms that produce nausea and vomiting, and finding treatments for these medical problems.

For this reason, we organized a conference that included scientists and clinicians from a variety of specialties with an interest in the biology and treatment of nausea and vomiting. The conference was held on October 3-4, 2013, on the campus of the University of Pittsburgh in Pittsburgh, Pennsylvania. Over 100 individuals attended the conference and traveled from as far away as Australia (Fig. 1; http://internationalvomitingconference.org). This meeting was supported in part by grant from the National Institute of Diabetes and Digestive and Kidney Disorders of the National Institutes of Health (NIH). A major success of the event was travel support provided to 11 early career investigators to present their research findings, which was possible because of NIH funding and an award from the Cyclic Vomiting Syndrome Association.

This issue of Experimental Brain Research contains 23 manuscripts related to presentations at the conference, including 9 reviews and 14 presentations of original data. Among the topics considered are the efficacy of different mechanisms in eliciting nausea and vomiting, assessing the severity of nausea, integration of neural signals by brain regions that coordinate nausea and vomiting, and treatment options for patients suffering from nausea and vomiting. 

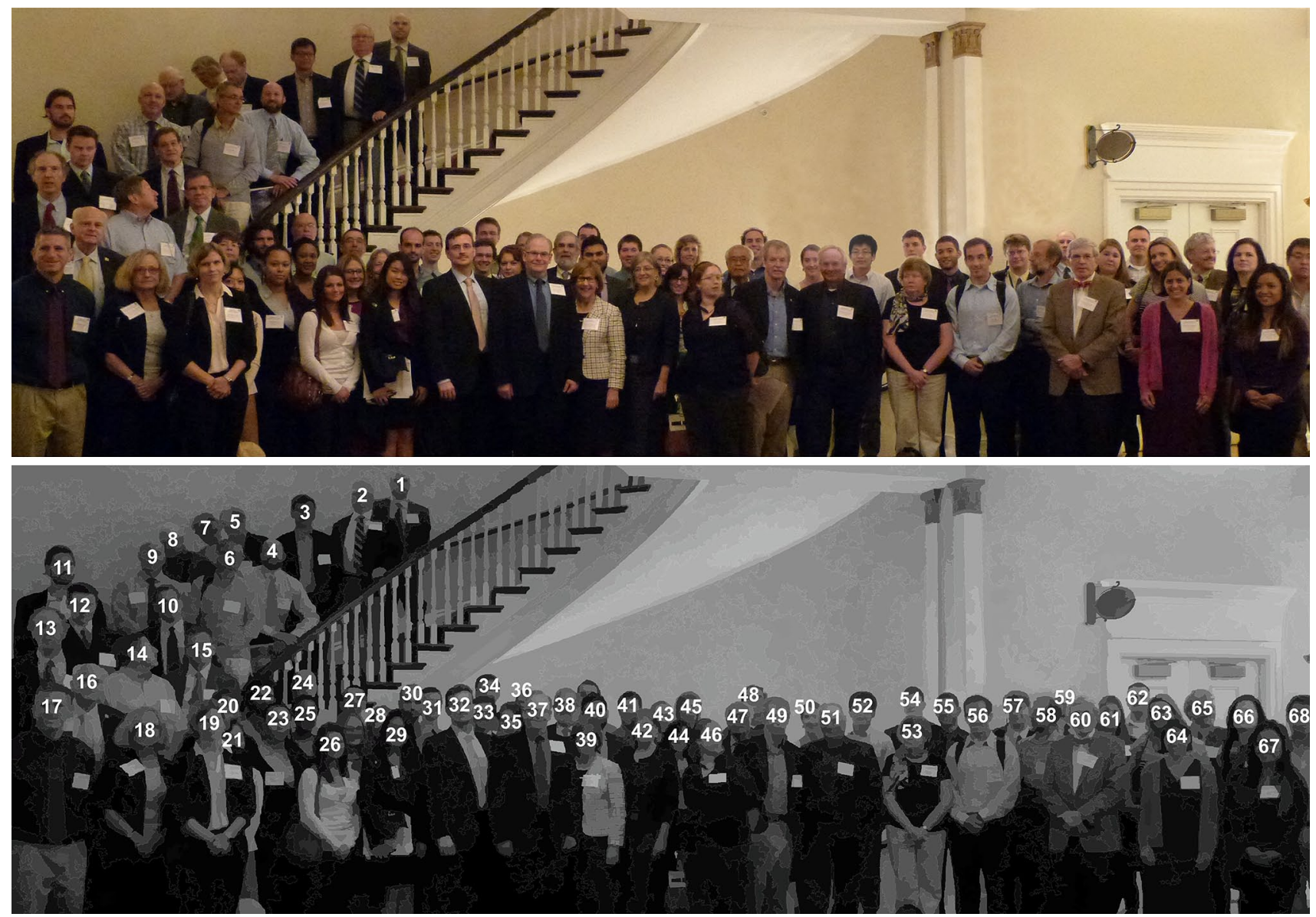

Fig. 1 Photograph of some the participants in the "Biology and Control of Nausea and Vomiting 2013" meeting. 1 Colin Ross, 2 Edwin Ross, 3 Braden Kuo, 4 David Levinthal, 5 Alan Miller, 6 Eugene Nalivaiko, 7 Kathleen Adams, 8 David Fleisher, 9 Lance Zirpel, 10 Richard Gralla, 11 Bart De Jonghe, 12 John Rudd, 13 Henry Parkman, 14 Jim Lucot, 15 Ken Koch, 16 Richard McCallum, 17 Max Levine, 18 Sally Tarbell, 19 Katja Kovacic, 20 Atiye Nur Aktay, 21 Doris Wong, 22 Behrang Keshavarz, 23 Ruby Holland, 24 Thomas Stoffregen, 25 Tanya H, Evans, 26 Megan Moore, 27 Camilo Rojas, 28 Linda Parker, 29 Audrey Lim, 30 Pierre Mourey, 31 David Rosenberg, 32 Charles Horn, 33 Nick Oberlies, 34 Samuel Dienel, 35 Per-

A strong consensus from the meeting was that additional multidisciplinary venues are needed to catalyze the scientific relationships required to understand the neural rine Deguilhen, 36 Laura Farr, 37 Bill Yates, 38 Carey Balaban, 39 Sue Wesmiller, 40 Baber Malik, 41 Christian Bohan, 42 Heidi Donovan, 43 Samantha Fortin, 44 Laura Rupprecht, 45 Linda Rinaman, 46 Kelly Meyers, 47 Etsuro Motoyama, 48 Matt Hayes, 49 Chuck Oman, 50 Alison Kreisler, 51 Gary Morrow, 52 Yi-Chou Chen, 53 Lucy Cotter, 54 Dan Miller, 55 Mike Catanzaro, 56 Bret Boyle, 57 Brian Williams, 58 Klaus Bielefeldt, 59 Tom Houpt, 60 Jim Lackner, 61 Hannah Kenward, 62 Martin Sticht, 63 Ann Marie King, 64 Marlena Fejzo, 65 Piotr Janicki, 66 Kimber MacGibbon, 67 Aromalyn Magtira, 68 Shigekazu Sugino

underpinnings of nausea and vomiting and to develop treatments for these conditions. Post-conference surveys indicated that the event was a major success (Fig. 2). Another 
Fig. 2 Results of the postmeeting survey of attendees of "Biology and Control of Nausea and Vomiting 2013". The electronic survey had a $59 \%$ response rate
To what extent were you satisfied with the overall quality of the conference?

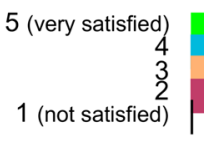

To what extent was the content of the program relevant to your practice and/or research?

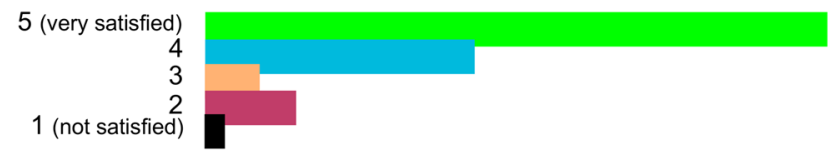

To what extent has your knowledge of the biological mechanisms for nausea and vomiting increased?

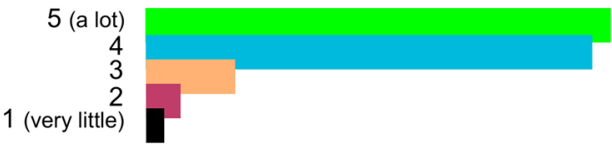

How often should the conference take place?

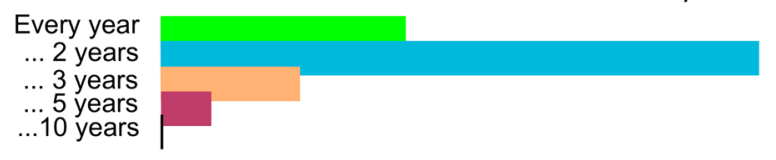

I was able to form new relationships that will help me in my research or clinical practice?

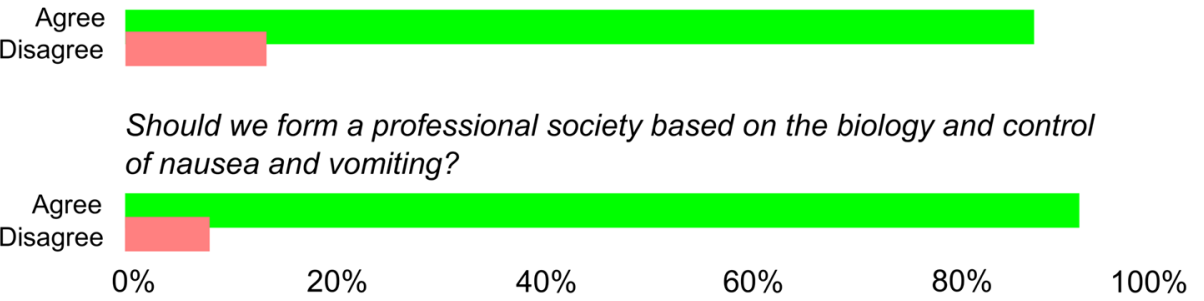

conference is planned for 2015, and efforts are underway to organize an academic society with a focus on nausea and vomiting.

Acknowledgments The conference "Biology and Control of Nausea and Vomiting 2013," which served as a catalyst for the publications in this issue of Experimental Brain Research, was sponsored by National Institutes of Health grant R13-DK100181, the Cyclic Vomiting Syndrome Association, Hyperemesis Education and Research (HER) Foundation, ProStrakan, and the Biobehavioral Oncology Program of the University of Pittsburgh Cancer Institute.

\section{References}

Lee LY, Abbott L, Mahlangu B, Moodie SJ, Anderson S (2012) The management of cyclic vomiting syndrome: a systematic review. Eur J Gastroenterol Hepatol 24:1001-1006. doi:10.1097/ MEG.0b013e328355638f

Stern RM, Andrews PLR, Koch KL (2011) Nausea: mechanisms and management. Oxford University Press, New York 